\title{
A Next Generation Imaging Filter for High Voltage Electron Microscopy
}

\author{
D. Moonen, C. Trevor, D. Chew, J. Hunt, P.E. Mooney, and H.A. Brink
}

Gatan Inc., 5933 Coronado Lane, Pleasanton, CA 94588, USA

We have designed and built a next generation high-voltage imaging filter (HV-GIF) which has several significant improvements with respect to the previous model.

Most present day TEMs work with acceleration voltages between 100 and $400 \mathrm{kV}$, but for some applications it is advantageous to use an electron beam with higher energy. Previously, Gubbens et al. ${ }^{[1]}$ reported on the design and testing of a post-column imaging filter suitable for use with electron-energies up to $1.25 \mathrm{MeV}$. Three of those filters were built and are still being used.

The mechanical design has been improved to make the system more rigid, reduce the number of vacuum seals, and optimize reliability. The original system consisted of a single magnetic prism with curved entrance and exit faces, 6 strong quadrupoles and 5 strong sextupoles after the prism and several weak lenses before the prism. The new filter includes additional multipole lenses to correct for non-symmetrical chromatic and geometric aberrations introduced by mechanical imperfections. Chromatic aberration correction is useful especially when the filter is operated at lower voltages. Typically, a high-voltage TEM is operated at several voltages with the lowest in the $400 \mathrm{kV}$ range.

The filter is equipped with three cameras. First, a 676 off-axis intensified TV-rate camera is located near the entrance of the filter. This camera can be operated independently from the filter itself. Second, a 692 retractable TV-rate camera with a phosphor scintillator is at the end of the imaging filter. This camera is mainly used to quickly find areas of interest in the specimen, or for alignment purposes. Third, a specially designed high-voltage MultiScan camera is installed behind the 692 TV-rate camera. This camera is used for the final acquisition of images and spectra. To prevent Xrays generated by the electron beam from hitting the CCD the image on the scintillator is reflected upwards using a mirror at an angle of $45^{\circ}$, and projected on the $1 \mathrm{k} \times 1 \mathrm{k} C C D$ with a custom-built projection system. ${ }^{[2]}$ The CCD can be adjusted with three degrees of freedom to focus the image.

This filter will be installed on a JEOL ARM1300S microscope, but can in principle be used with any high-voltage microscope.

\section{References}

[1] A.J. Gubbens et al., Ultramicroscopy 59 (1995) 255-256.

[2] P.E. Mooney et al., Proc. ICEM 13 (1994) 213-214. 

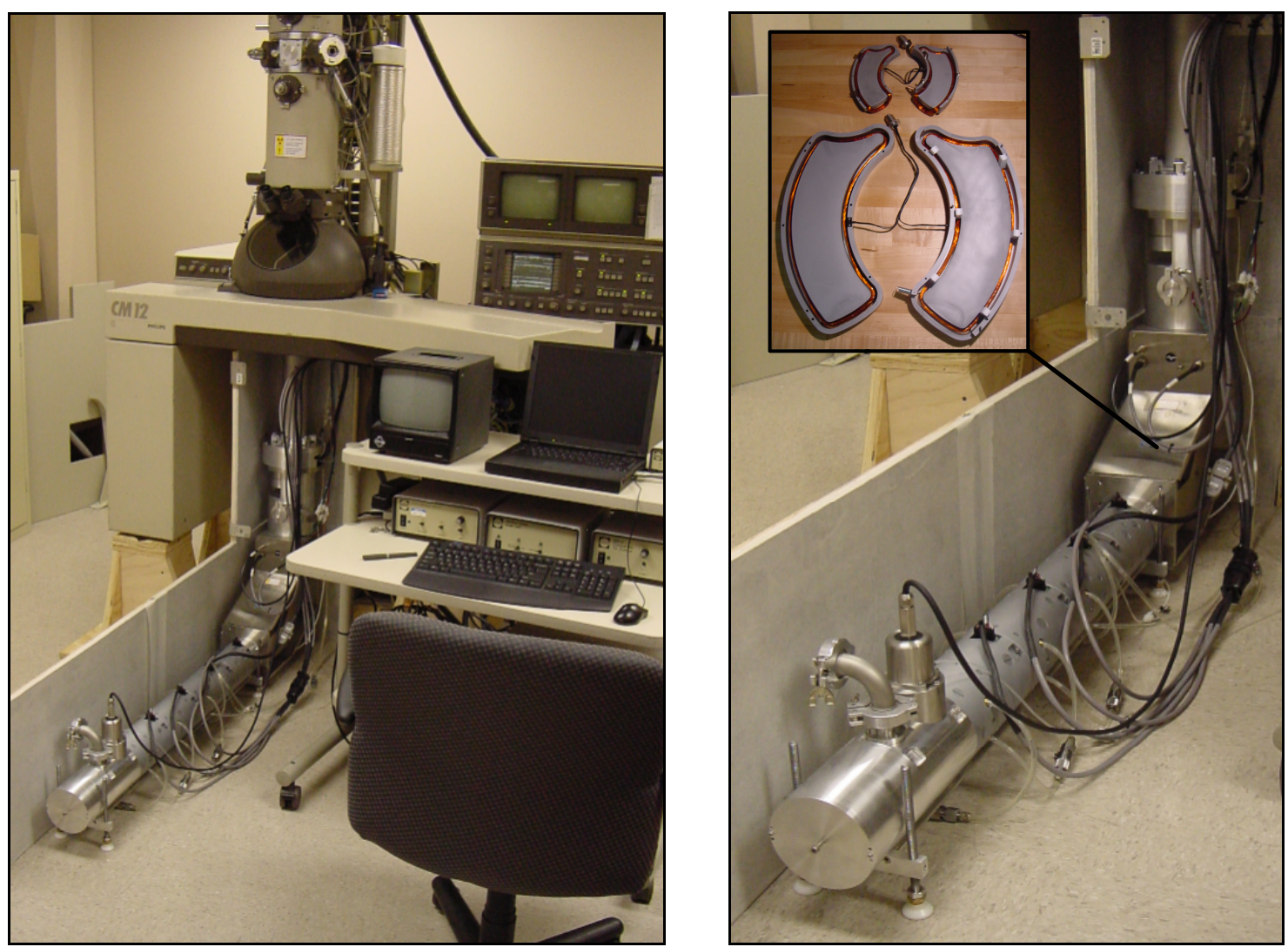

FIG 1. The HV-GIF installed on a Philips CM12 TEM for initial testing. The TEM was raised 50 $\mathrm{cm}$ above floor level. Insert: comparison of the prism magnet of a regular post-column imaging filter (top) and for the HV-GIF (bottom).

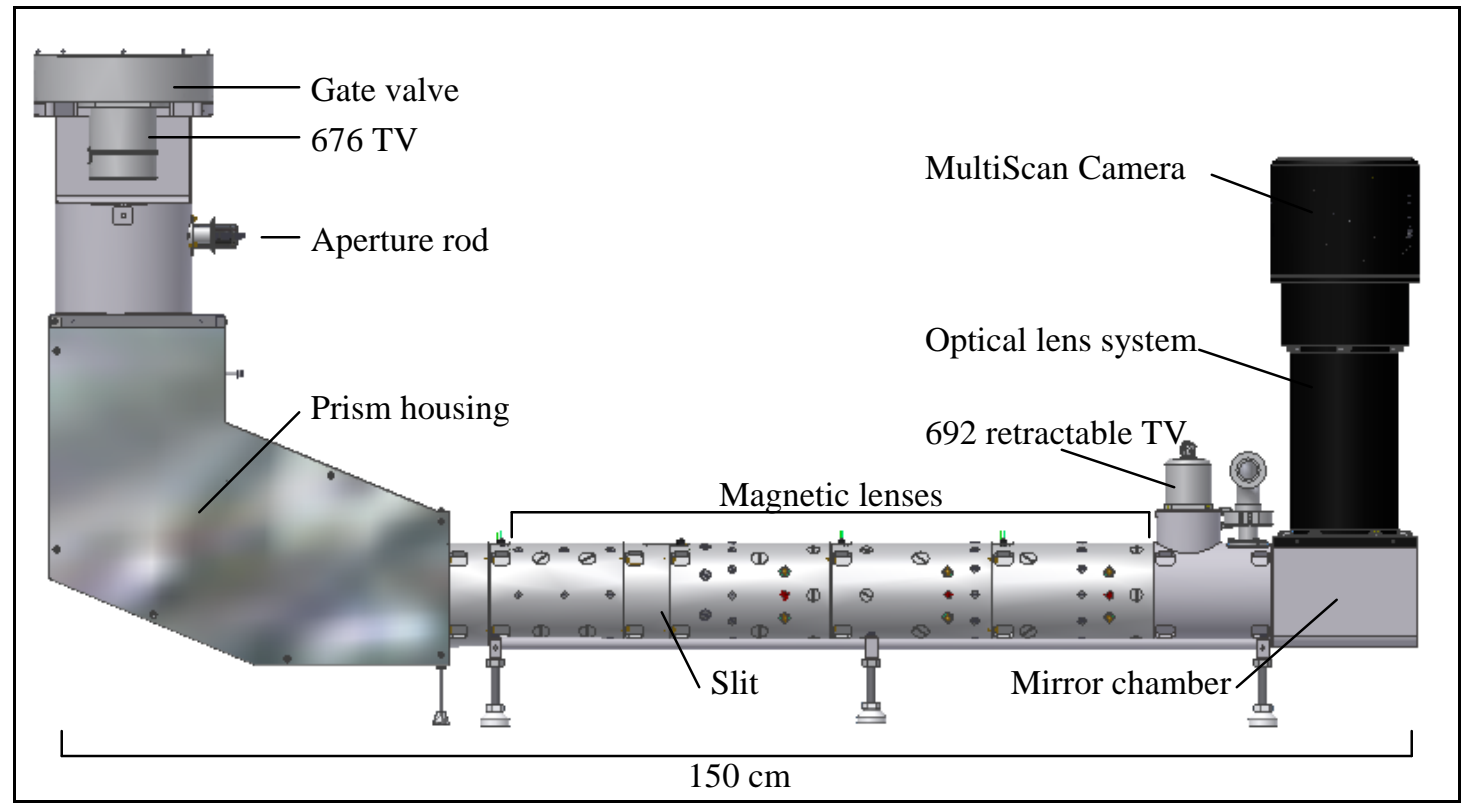

FIG 2. Drawing of the HV-GIF with two TV-rate cameras and the new HV MultiScan camera. 